\title{
Clinical Experience of Antifungal Combination Therapy for Invasive Fungal Infections in Pediatric Hematological Malignancy Patients
}

\author{
Saliha Kanık Yüksek ${ }^{1}$, Aslınur Özkaya Parlakay ${ }^{1}$, Belgin Gülhan ${ }^{1}$, Neşe Yaralı ${ }^{1}$, Namık \\ Yaşar Özbek ${ }^{1}$, and Hasan Tezer ${ }^{2}$ \\ ${ }^{1}$ Ankara City Hospital \\ ${ }^{2}$ Gazi University Faculty of Medicine
}

January 20, 2021

\begin{abstract}
Background: The role of combination regimens in the treatment of invasive fungal infections (IFI) in patients with hematologic malignancies remains unclear. This study was aimed to demonstrate experience data about combined antifungal therapy (CAT) in pediatric IFI patients with haematological malignancies. Methods: Between January 2014 and December 2017, a total of 33 IFI episodes in 28 patients with hematological malignancies were analyzed retrospectively. Results: Of the patients (19 with acute lymphoblastic leukemia, and 9 with acute myeloblastic leukemia), 21 (75\%) had leukemia relapse and 7 (25\%) had remission. IFI was classified as possible in 26 (78.8\%) episodes, probable in $5(15.1 \%)$ episodes, and proven in $2(6.1 \%)$ episodes. LamB (\%50) was the most preferred agent in monotherapy. Mean duration of monotherapy was $12.84 \pm 4.28$ (5-24) days. LamB plus voriconazole $(54.5 \%)$ were the most common combination preference in CAT. Mean duration of CAT was $42.36 \pm 36.4$ days, and unchanged according to combination regimen type $(\mathrm{p}=0.571)$. Total mortality rate and IFI attributable mortality rate were $60.7 \%$ vs $76.5 \%$. Mortality rate was significantly higher in patients with relapse $(\mathrm{p}=0.006)$. Complete response was obtained in $81.8 \%$ of surviving patients. Duration of neutropenia and CAT, and recovery time were not found statistically different in the episodes with/without death and according to relapse or remission status. Side effects due to CAT use were observed quite low level. Conclusion: CAT has been found to be safe in IFI episodes of pediatric leukemia. The result will contribute to the data about combined antifungal use in daily clinical practice in pediatric haematological patients with IFI.
\end{abstract}

\section{Clinical Experience of Antifungal Combination Therapy for Invasive Fungal Infections in Pe- diatric Hematological Malignancy Patients}

Saliha Kanik-Yuksek ${ }^{1}$, Aslınur Özkaya Parlakay ${ }^{1}$, Belgin Gülhan ${ }^{1}$, Neşe Yaralı ${ }^{2}$, Namık Yaşar Özbek ${ }^{2}$, Hasan Tezer $^{3}$

${ }^{1}$ University of Health Sciences, Ankara City Hospital, Pediatric Infectious Diseases Department, Turkey

${ }^{2}$ University of Health Sciences, Ankara City Hospital, Department of Pediatric Hematology Oncology

${ }^{3}$ Gazi University Medical Faculty, Pediatric Infectious Diseases Department, Turkey

Correspondence: Saliha Kanık Yüksek, MD

University of Health Sciences, Ankara City Hospital, Department of Pediatric

Infectious Diseases

Address: Üniversiteler Mahallesi 1604. Cadde No: 9, 06800, Çankaya, Ankara, Turkey 
Telephone: +903125526000

\section{E-mail:}

salihakanik@gmail.com

\section{Abstract}

Background: The role of combination regimens in the treatment of invasive fungal infections (IFI) in patients with hematologic malignancies remains unclear. This study was aimed to demonstrate experience data about combined antifungal therapy (CAT) in pediatric IFI patients with haematological malignancies.

Methods: Between January 2014 and December 2017, a total of 33 IFI episodes in 28 patients with hematological malignancies were analyzed retrospectively.

Results: Of the patients (19 with acute lymphoblastic leukemia, and 9 with acute myeloblastic leukemia), 21 $(75 \%)$ had leukemia relapse and $7(25 \%)$ had remission. IFI was classified as possible in $26(78.8 \%)$ episodes, probable in $5(15.1 \%)$ episodes, and proven in $2(6.1 \%)$ episodes. LamB (\%50) was the most preferred agent in monotherapy. Mean duration of monotherapy was $12.84 \pm 4.28$ (5-24) days. LamB plus voriconazole (54.5\%) were the most common combination preference in CAT. Mean duration of CAT was $42.36 \pm 36.4$ days, and unchanged according to combination regimen type $(p=0.571)$. Total mortality rate and IFI attributable mortality rate were $60.7 \%$ vs $76.5 \%$. Mortality rate was significantly higher in patients with relapse $(p=$ 0.006). Complete response was obtained in $81.8 \%$ of surviving patients. Duration of neutropenia and CAT, and recovery time were not found statistically different in the episodes with/without death and according to relapse or remission status. Side effects due to CAT use were observed quite low level.

Conclusion: CAT has been found to be safe in IFI episodes of pediatric leukemia. The result will contribute to the data about combined antifungal use in daily clinical practice in pediatric haematological patients with IFI.

Keywords: Pediatric; Leukemia; Invasive fungal infection; Combined antifungal therapy

\section{What is already known about this topic?}

- Monotherapy with antifungal agents is often preferred for the treatment of invasive fungal infections (IFI) in pediatric patients, however the combination of antifungals remains on the agenda as a potential treatment strategy where monotherapy is insufficient.

- The efficacy and role of combination regimens in the treatment of IFI in hematologic malignancies patients remains controversial.

- Previous studies of pediatric IFI are quite inadequate, and most knowledge regarding the treatment of IFI is gathered from studies in adults. What does this article add?

- The clinical experience data on the use of CAT in pediatric patients with haematological malignancies with IFI, including the results of efficacy and toxicity, were presented here.

- This study is remarkable considering the difficulties in planning studies in pediatric patient group, limited data about IFI treatment for clinical use, and no definitive accepted recommendation.

\section{Introduction}

Although many and novel antifungal agents are in use, invasive fungal infections (IFI) continue to cause high morbidity and mortality rates in pediatric hematologic malignancy patients. ${ }^{1,2}$ Successful treatment of IFI is required to ensure the survival of this population. Currently, there are four classes (azoles, polyenes, pyrimidine analogues, and echinocandins) of drugs used in the treatment of IFI in children. ${ }^{3}$ Appropriate use of existing antifungals in this vulnerable population is important for the treatment of IFI. ${ }^{2}$ However, safety and efficacy data including antifungal activity, pharmacokinetic properties, and toxicity of the antifungal agents in children still needs to be supported by trials. ${ }^{3,4}$ Monotherapy is often preferred for the treatment of fungal infections in pediatric patients. ${ }^{4}$ In some serious fungal infections where monotherapy is insufficient, the combination of antifungals remains on the agenda as a potential treatment strategy. ${ }^{5}$ Because serious 
fungal infections need to be cured during the treatment of primary diseases, children with hematologic malignancies are the group of patients in whom combination regimens are frequently considered and tried to be applied. ${ }^{4,5}$

Antifungal combination therapies are used in hematologic malignancy patients considering potential gains such as preventing resistance problems, increasing treatment efficacy and reducing side effects. ${ }^{5,6}$ Combined antifungal therapy (CAT) is not a new notion, it is even used effectively in the treatment of some welldefined infections. ${ }^{7}$ Unfortunately, the role of combination regimens in the treatment of IFI in patients with hematologic malignancies remains controversial. ${ }^{8}$ Despite insufficient evidence, there are pre-clinical studies indicating that combination regimens are effective in fungal infections difficult to treat. ${ }^{9-11}$ However, these studies could not be transferred to the clinical practice, and quite few data are available for clinical use. The limited data on this treatment prescription, with almost no prospective studies and more limited data in pediatric patients, are obtained as a result of clinical experience. ${ }^{6,8,12-15}$ The combinations of four groups of antifugal agents, acting through different molecular pathways and different cellular targets, is shaped to the preference and priority of clinicians as there is no definitive accepted recommendation. ${ }^{8,9}$ In the absence of sufficient evidence and suggestions; this study was aimed to demostrate experience data on the use of CAT in pediatric patients with haematological malignancies with IFI, including the results of efficacy and toxicity.

\section{Materials and Methods}

A retrospective study was conducted at 'Health Science University Ankara Children's Hematology Oncology Training and Research Hospital' from January 2014 until December 2017. Patients under 18 years of age with a hematological malignancy (acute lymphoblastic or myeloblastic leukemia) diagnosed as IFI were enrolled. Basic demographic data, underlying conditions, neutropenia (absolute neutrophil count $<500$ cells $/ \mu \mathrm{L}$ ) durations, radiological findings, antifungal medications and side effects, and outcomes were noted. Galactomannan (GM) enzyme immunoassay (Platelia Aspergillus, BioRad, France) analysis in plasma were obtained in the presence neutropenic fever and IFI episode twice weekly, and [?]0.5 were considered positive. All patients received prophylactic antifungal agents according to their primary disease protocol, and the risk or history of IFI. Diagnosis of IFI was defined acording to the criteria of the European Organization for Research and Treatment of Cancer/Invasive Fungal Infections Cooperative Group and the National Institute of Allergy and Infectious Disease Mycoses Study Group (EORTC/MSG). ${ }^{16}$ Patients were grouped according to a diagnosis of proven, probable, or possible IFI. Empirical antifungal treatment was initiated if fever persisted for more than 96 hours after initiation of empiric antibacterial treatment considering guidelines. ${ }^{17,18}$ If there were symptoms, signs, laboratory or radiological findings suggestive of invasive fungal infection, antifungal therapy was initiated earlier than the above-mentioned period. CAT was initiated based on the patient's IFI condition and clinician's subjective decision. The day of diagnosis was defined as the day that the clinician confirmed diagnosis of IFI, and onset the antifungal treatment. Response to treatment was defined according to criteria of EORTC/MSG. ${ }^{16}$ Overall response rate and overall survival rate based on 12 weeks were recorded. Research ethics board approval was obtained for the study from the local ethics committee of institution.

Statistical analysis was performed using SPSS v25.0 (IBM Corp., Armonk, New York, USA) statistical package. Categorical variables were compared by chi-square or Fisher exact tests, and were summarized with frequencies. For continuous variables, median and interquartile range (IQR) were calculated. Group comparisons were carried out using independent samples t-test or Mann-Whitney U-tests, and Kruskal-Wallis test if appropriate. All tests were 2-sided with a significance level of 0.05.

\section{Results}

Thirty three IFI episodes in 28 patients were examined. Two or more episodes were recorded in four patients. Demographic and clinical characteristics of the patients, and data for IFI were listed inTable 1 . None of the patients underwent hematopoietic stem cell transplantation prior to IFI onset. GM analysis was applied to all patients at the beginning of a febrile neutropenia attack and in the continuation in necessary patients, and was positive in $10(32.3 \%)$ episodes. The mean GM index of the positive episodes was $3.27 \pm 3.35(0.74-9)$. 
The most common radiological findings were ground-glass view (75.8\%), nodules (54.5\%) and consolidations (24.3\%). Cavitation and/or halo sign were recorded in 7 episodes. All 27 episodes that were detected any radiological finding were pulmonary IFI; the remainder were hepatosplenic (in 2 episodes), central nervous system (in 1 episode), and paranasal (in 1 episode) involvement, respectively.

Liposomal amphotericin B (LAmB) was the first antifungal agent for monotherapy in 16 (50\%) of IFI episodes, caspofungin in $11(34.4 \%)$, and voriconazole in 5 (15.6\%) episodes. Mean duration of monotherapy was $12.84 \pm 4.28$ (5-24) days. The second antifungal was added because of insufficient response at all episodes, and preferences were listed as follows: voriconazole in $22(68.7 \%)$ patients, LAmB in $7(21.8 \%)$ patients, caspofungin in $2(6.4 \%)$ patients, and posaconazole in $1(3.2 \%)$ patient. CAT was initiated directly in only one episode as voriconazole plus caspofungin. Preferred combination regimes; duration of therapy, treatment responses, and mortality rates were summarized inTable 2 . Range for treatment duration with combined antifungal was 15-67 days, and the range of transition day from combined regimen to monotherapy in surviving patients was 28-67 days. The effect of the combination regimen type on mortality was found to be statistically insignificant $(\mathrm{z}=1.3 ; p=0.192)$. In both of the proven IFI episodes, the causative agents were Candida spp .

The 12 -week and overall survival rate of the patients was $75 \%$ and $39.2 \%$, repectively. The cause of death was IFI progression in 13 of the 17 patients, and the remaining patients had non-responsiveness to underlying disease treatment. IFI attributable mortality rate was $76.5 \%$. Mortality rate was significantly higher in patients with relapse $\left(\chi^{2}=7.47 ; p=0.006\right)$. Complete response $(\mathrm{CR})$ to the treatment was obtained in 9 $(81.8 \%)$ of 11 surviving patients. Mean recovery time in patients with CR was $96.45 \pm 57.95$ (48-210) days. Neutropenia duration $(\mathrm{z}=0.39 ; p=0.695)$, duration of CAT $(\mathrm{z}=1.37 ; p=0.173)$, and recovery time $(\mathrm{z}$ $=0.768 ; p=0.443)$ were not found statistically different in the episodes with/without death. The effect of the selected primary antifungal agent could not be evaluated due to insufficient number of cases. Duration of neutropenia $(\mathrm{z}=0.22 ; p=0.821)$ and CAT $(\mathrm{t}=0.795 ; p=0.446)$, and recovery time $(\mathrm{t}=0.991 ; p=$ $0.355)$ were also not statistically different according to relapse or remission status. There was no statistically significant relationship between IFI classification and the episodes with/without death $\left(\chi^{2}=3.726 ; p=\right.$ 0.293). Among the surviving patients, voriconazole was preferred in 9 patients as the secondary antifungal prophylactic agent, and posaconazole in two patients.

CAT was well tolerated in most patients. Renal dysfunction accompanied by electrolyte disturbances in one patient and increased transaminases in two patients were seen as adverse effects of CAT, and treatment discontinuation was required in only one patient because of side effects. Plasma levels monitoring for voriconazole was not provided since not implemented in our center. IFI-induced surgical procedure was not applied to any patient.

\section{Discussion}

IFI is an important condition in patients with hematological malignancies due to devastating effects. ${ }^{5}$ Despite timely diagnosis and treatment, IFI can result in serious morbidity and mortality. ${ }^{1}$ Previous studies of pediatric IFI are quite inadequate, and most knowledge regarding the treatment of IFI is gathered from studies in adults. ${ }^{16,17}$ Because of the difficulty of performing prospective studies in this vulnarable patient group, data were obtained retrospectively in almost all of the limited number of studies. ${ }^{17}$ Considering the difficulties in planning studies in pediatric patient group and limited data about IFI treatment, the value of any study that contributes to the literature is obvious. This study is remarkable in the light of the limitations that have been accepted in this regard.

IFI-related mortality rates range from $45 \%$ to $90 \%$, and treatment success for IFI is reported to be around $60 \%$ in various reports. ${ }^{1,5,19,20}$ Since the advantages and disadvantages have not been determined compared to monotherapy, CAT in IFI is still unclear. However, thanks to increased drug synergy and efficacy, and decreased resistance to antifungal drugs, the expectation that CAT may improve the outcome of the patients occurs. Due to the absence of prospective and randomized controlled clinical trials with an adequate statistical power, combined antifungal use in IFI treatment is included in current international guidelines as 
a low level of evidence and recommendation. ${ }^{17,18}$ Although the CAT approach in IFI has weak foundations, it is frequently applied in daily practice ${ }^{14}$, and there are even reports emphasizing that this choice has reached $90 \% .^{21}$

In previously published studies, overall response to CAT was reported at rates ranging from $35 \%$ to $60 \%$ (8). The 12 -week survival rate was generally reported at higher than the overall response rate. ${ }^{8-12}$ Previous studies reported that CAT appears helpful especially in patients with poor prognostic features. ${ }^{8}$ In our results, considering that patients generally consist of children with poor prognostic factors such as prolonged neutropenia and relapse leukemia, the overall response to CAT can be interpreted as good although there is no control group. However, ultimately the mortality rate attributed to IFI was found to be quite high. Neutropenia is an important risk factor for IFI, and the duration of neutropenia affects the treatment response and mortality rates. ${ }^{2}$ In our patient group, the mean duration of neutropenia was found to be $42.87 \pm 35.5$ days, and this neutropenia duration was detected longer than previous studies. ${ }^{19,22}$ Although the duration of neutropenia was not statistically different in the episodes with/without death, was the main determinant in the high IFI attributable mortality rate, as our opinion.

In our results, LamB was found to be the most preferred first line agent in monotherapy due to the inability to distinguish invasive aspergillosis and mucormycosis on the day of diagnosis. Monotherapy was switched to CAT in approximately two weeks in all episodes and voriconazole-based combination regimen was applied most frequently. Although in vitro studies have reported that caspofungin and voriconazole have a synergistic effect against Aspergillus spp ${ }^{23,24}$ and this combination reduces fungal burden in animal tissues compared to single echinocandin or triazole administration ${ }^{11}$, the combination regimen preferences may vary in practice. ${ }^{8,14,15}$ In our experience, the choice of antifungal combination was found to vary according to the first antifungal chosen in monotherapy, and LamB plus voriconazole were the most common preference. The total mortality rate in combination regimens was around $60 \%$ in our patients, and the highest mortality rate was seen in the voriconazole plus caspofungin combination with $80 \%$. When evaluating this result, it should be kept in mind that there is not a patient group receiving monotherapy for comparison, and there was not a control group. In response to the expectation of improvement in patients' outcomes, there are reports claimed that CAT is ineffective in general patient outcomes ${ }^{6,14,22,25}$, despite clinical trials with positive results. ${ }^{6,12}$ In our study, CAT could not be compared with monotherapy due to absence of a control group, but the combination regimen type was found to be ineffective on mortality. This study demonstrated once again that the uncontrolled underlying disease was one of the most important factors affecting the mortality rate. ${ }^{1,2}$

When the previous studies are evaluated, it is seen that both in the transition time from monotherapy to CAT and in the duration of CAT do not have a standard, and varies widely. ${ }^{6,8,10,12-15}$ In this study, the mean duration of therapy was $42.36 \pm 36.4$ days, and unchanged according to combination regimen type. The main determinant of the duration of CAT seems to be the combination of clinical, radiological and microbiological response of the patient. Due to individual differences in determining the treatment reflection, CAT durations can be very short or very long. ${ }^{6,12-15}$

CAT was generally reported as associated with an increased risk of adverse events. ${ }^{14,22}$ Although it variables according to the type of antifungal drug used, the most common side effects of CAT were reported as liver, renal, and neurologic toxicity. ${ }^{12,13}$ Despite reports indicating increased risk of side effects, there are also studies that proved CAT does not cause significant side effects other than mild or moderate events. ${ }^{6,26}$ In our experience, CAT resulted as well tolerated generally. Namely, as a positive result, CAT has been found to be safe in IFI episodes of pediatric leukemia.

This study has some limitations. Our study is a retrospective study reporting data from only one institute. Due to the small number of patients, the power of statistical comparisons decreased. Furthermore, due to quite low number of proven IFI, it is not clear which combined antifungal regimen is administered for IFI, which fungal agent is caused. Despite the limitations, we think that the data of this study may contribute to the analysis of combined antifungal use in daily clinical practice, tolerability and results in haematological patients with IFI. 
Optimal therapy for IFI in pediatric hematological malignancy patients is unknown, some clinicians use the CAT approach as an alternative choice to improve the outcome of these critically ill patients. However, while various pre-clinical studies suggest the possibility of this preference, there is no definite accepted recommendation yet. Well-designed and randomized trials are required to define the role of combined antifungal use in pediatric haematological patients.

Disclosure: The authors declare no conflict of interests.

Funding: The author(s) received no financial support for the research, authorship, and/or publication of this article.

\section{References}

1. Cesaro S, Tridello G, Castagnola E, et al. Retrospective study on the incidence and outcome of proven and probable invasive fungal infections in high-risk pediatric onco-hematological patients.Eur J Haematol. 2017;99(3):240-248. doi: 10.1111/ejh.12910

2. Kobayashi R, Hori D, Sano H, Suzuki D, Kishimoto K, Kobayashi K. Risk Factors for Invasive Fungal Infection in Children and Adolescents With Hematologic and Malignant Diseases: A 10-year Analysis in a Single Institute in Japan. Pediatr Infect Dis J.2018;37(12):1282-1285.

3. Puia-Dumitrescu M, Smith PB. Antifungal Drugs in Newborns and Children. Pediatr Clin North Am. 2017;64(6):1389-1402. doi: 10.1016/j.pcl.2017.08.013.

4. Ramos-Martín V, O'Connor O, Hope W. Clinical pharmacology of antifungal agents in pediatrics: children are not small adults. Curr Opin Pharmacol. 2015;24:128-34. doi: 10.1016/j.coph.2015.08.009.

5. Pagano L, Caira M, Valentini CG, Posteraro B, Fianchi L. Current therapeutic approaches to fungal infections in immunocompromised hematological patients. Blood Rev. 2010;24(2):51-61. doi: 10.1016/j.blre.2009.11.003.

6. Candoni A, Caira M, Cesaro S, et al; SEIFEM GROUP (Sorveglianza Epidemiologica Infezioni Fungine nelle Emopatie Maligne). Multicentre surveillance study on feasibility, safety and efficacy of antifungal combination therapy for proven or probable invasive fungal diseases in haematological patients: the SEIFEM real-life combo study.Mycoses. 2014;57(6):342-50. doi: 10.1111/myc.12161.

7. Day JN, Chau IT, Lalloo DG. Combination antifungal therapy for Cryptococcal Meningitis. $N$ Engl $J$ Med. 2013;368:2522-3.

8. Mihu CN, Kassis C, Ramos ER, Jiang Y, Hachem RY, Raad II. Does combination of lipid formulation of amphotericin B and echinocandins improve outcome of invasive aspergillosis in hematological malignancy patients? Cancer. 2010;116(22):5290-6. doi: 10.1002/cncr.25312.

9. MacCallum DM, Whyte JA, Odds FC. Efficacy of caspofungin and voriconazole combinations in experimental aspergillosis. Antimicrob Agents Chemother. 2005;49:3697-701.

10. Ibrahim AS, Gebremariam $\mathrm{T}, \mathrm{Fu} \mathrm{Y}$, et al. Combination echinocandin polyene treatment of murine mucormycosis. Antimicrob Agents Chemother. 2008;52:1556-8.

11. Kirkpatrick WR, Coco BJ, Patterson TF. Sequential or combination antifungal therapy with voriconazole and liposomal amphotericin B in a guinea pig model of invasive aspergillosis.Antimicrob Agents Chemother. 2006;50:1567-9.

12. Cesaro S, Giacchino M, Locatelli F, Spiller M, Buldini B, Castellini C, et al. Safety and efficacy of a caspofungin-based combination therapy for treatment of proven or probable aspergillosis in pediatric hematological patients. BMC Infect Dis. 2007;7:28.

13.Raad II, Zakhem AE, Helou GE, Jiang Y, Kontoyiannis DP, Hachem R. Clinical experience of the use of 
voriconazole, caspofungin or the combination in primary and salvage therapy of invasive aspergillosis in haematological malignancies. Int J Antimicrob Agents.2015;45(3):283-8. doi: 10.1016/j.ijantimicag.2014.08.012.

14. Wattier RL, Dvorak CC, Hoffman JA,Brozovich AA, Bin-Hussain I, Groll AH, et al. A Prospective, International Cohort Study of Invasive Mold Infections in Children. J Pediatric Infect Dis Soc.2015;4(4):31322. doi: $10.1093 /$ jpids/piu074

15.Marr KA, Schlamm HT, Herbrecht R, Rottinghaus ST, Bow EJ, Cornely OA, et al. Combination antifungal therapy for invasive aspergillosis: a randomized trial. Ann Inern Med. 2015;162(2):81-9. doi: 10.7326/M132508 .

16. Segal BH, Herbrecht R, Stevens DA, et al. Defining responses to therapy and study outcomes in clinical trials of invasive fungal diseases: Mycoses Study Group and European Organization for Research and Treatment of Cancer consensus criteria. Clin Infect Dis. 2008;47:674-83.

17. Groll AH, Castagnola E, Cesaro S, et al. Fourth European Conference on Infections in Leukaemia (ECIL4): guidelines for diagnosis, prevention, and treatment of invasive fungal diseases in paediatric patients with cancer or allogeneic haemopoietic stem-cell transplantation. Lancet Oncol. 2014;15:e327-40.

18.Patterson TF, Thompson GR 3rd, Denning DW, Fishman JA, Hadley S, Herbrecht R, et al. Practice Guidelines for the Diagnosis and Management of Aspergillosis: 2016 Update by the Infectious Diseases Society of America. Clin Infect Dis. 2016;63(4):e1-e60. doi: 10.1093/cid/ciw326.

19. Lee KH, Lim YT, Hah JO, Kim YK, Lee CH, Lee JM. Voriconazole plus caspofungin for treatment of invasive fungal infection in children with acute leukemia.Blood Res. 2017;52(3):167-173.

20. Kurosawa M, Yonezumi M, Hashino S, et al. Epidemiology and treatment outcome of invasive fungal infections in patients with hematological malignancies. Int $J$ Hematol. 2012;96:748-57.

21. Georgiadou SP, Pongas G, Fitzgerald NE, Lewis RE, Rytting M, Marom EM, Kontoyiannis DP. Invasive Mold Infections in Pediatric Cancer Patients Reflect Heterogeneity in Etiology, Presentation, and Outcome: A 10-Year, Single-Institution, Retrospective Study.J Pediatric Infect Dis Soc. 2012 Jun;1(2):125-35. 22. Raad II, Zakhem AE, Helou GE, Jiang Y, Kontoyiannis DP, Hachem R. Clinical experience of the use of voriconazole, caspofungin or the combination in primary and salvage therapy of invasive aspergillosis in haematological malignancies.Int J Antimicrob Agents. 2015;45(3):283-8.

23. Perea S, Gonzalez G, Fothergill AW, Kirkpatrick WR, Rinaldi MG, Patterson TF. In vitro interaction of caspofungin acetate with voriconazole against clinical isolates of Aspergillus spp.Antimicrob Agents Chemother. 2002;46:3039-41.

24. Petraitis V, Petraitiene R, Sarafandi AA, Kelaher AM, Lyman CA, Casler HE, et al. Combination therapy in treatment of experimental pulmonary aspergillosis: synergistic interaction between an antifungal triazole and an echinocandin. J Infect Dis. 2003;187:1834-43.

25. Marr KA, Boeckh M, Carter RA, Kim HW, Corey L. Combination antifungal therapy for invasive aspergillosis. Clin Infect Dis.2004;39:797-802.

26. Groll AH, Silling G, Young C, Schwerdtfeger R, Ostermann H, Heinz WJ, et al. Randomized comparison of safety and pharmacokinetics of caspofungin, liposomal amphotericin $\mathrm{B}$, and the combination of both in allogeneic hematopoietic stem cell recipients. Antimicrob Agents Chemother.2010;54(10):4143-9. doi: 10.1128/AAC.00425-10.

Table 1. Demographic and clinical characteristics of the patients, and data for IFI episodes.

\begin{tabular}{ll}
\hline Total cases & 28 \\
Age (years) Mean \pm SD Median (range) & $8.79 \pm 5.037(3.5-18)$ \\
Gender, $\mathbf{n}(\%)$ Male Female & $15(53.6 \%) 13(46.4 \%)$ \\
Underlying disease, n (\%) ALL AML & $19(67.9 \%) 9(32.1 \%)$
\end{tabular}


Status of underlying disease, $\mathrm{n}(\%)$

Remission Relaps

Prophylactic agent before IFI onset, n (\%)

Flucanazole Voriconazole

Prophylaxis duration before IFI episodes

(days) Mean \pm SD (range)

Duration of neutropenia at IFI onset

(days)* Mean $\pm \mathrm{SD}$ (range) in ALL in AML

IFI classification, episode (\%) Possible

Probable Proven
$7(25 \%) 21(75 \%)$

$26(92.8 \%) 2(7.2 \%)$

$47 \pm 24.04(30-84)$

$42.87 \pm 35.5(7-140) 42.18 \pm 40.1(7-140) 44.75 \pm$

$20(20-64)$

$26(78.8 \%) 5(15.1 \%) 2(6.1 \%)$

*No statistical difference was found according to the underlying disease $(p=0.18)$

ALL: Acute lymphoblastic leukemia, AML: Acute myeloblastic leukemia, IFI: Invasive fungal infection, SD: Standart deviation,

Table 2. Preferred combination regimes; duration of therapy, treatment responses, and mortality rates.

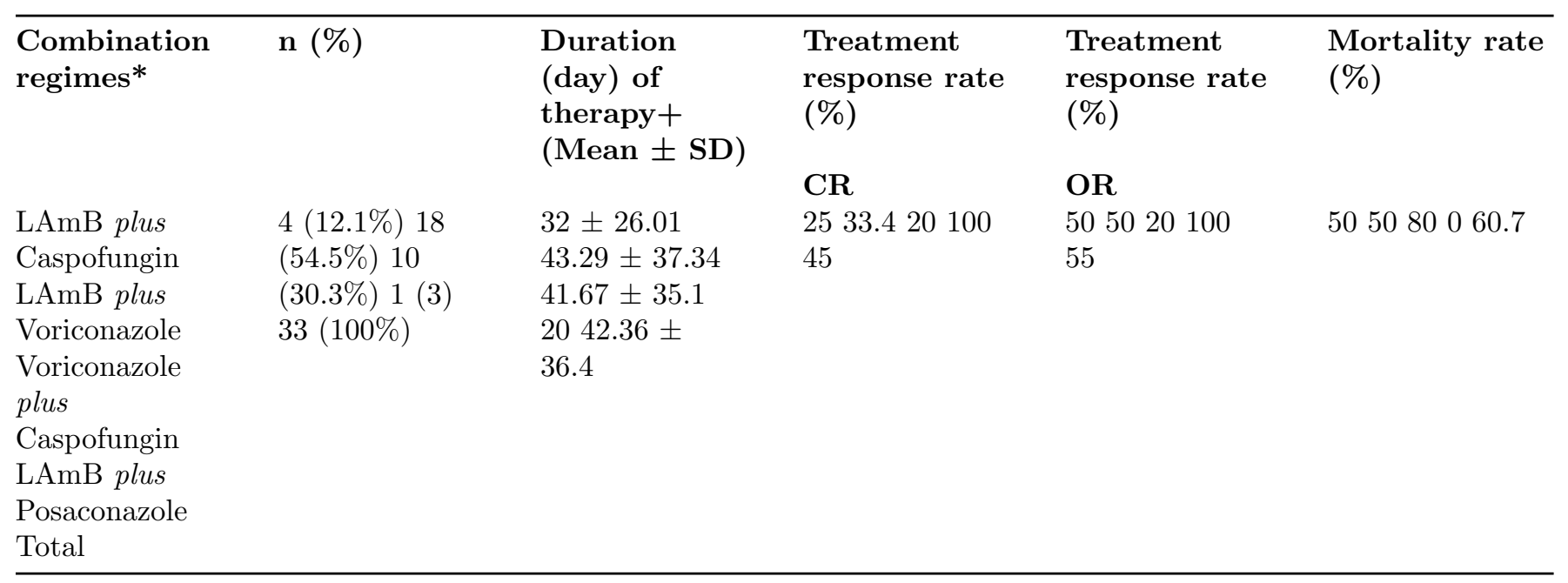

CR: Complete response, LAmB: Liposomal amphotericin B, OR: Overall response

*All antifungals applied during CAT were used at the recommended doses for monotherapy.

+was not statistically significant according to the combination regimen $(p=0.571)$. 